\title{
Increased cancer-specific mortality of very small size in carcinoembryonic antigen-elevated rectal cancer
}

\author{
Haiqiang Pan $^{1}$, Junhui Cui ${ }^{1}$, Ke Cai ${ }^{1}$, Yena Zhou ${ }^{2}$ \\ ${ }^{1}$ Department of Colorectal Surgery, ${ }^{2}$ Department of General Surgery, Tongde Hospital of Zhejiang Province, Hangzhou 310012, China \\ Contributions: (I) Conception and design: H Pan, Y Zhou; (II) Administrative support: K Cai, Y Zhou; (III) Provision of study materials or patients: \\ H Pan, J Cui, K Cai; (IV) Collection and assembly of data: H Pan, J Cui; (V) Data analysis and interpretation: H Pan; (VI) Manuscript writing: All \\ authors; (VII) Final approval of manuscript: All authors. \\ Correspondence to: Yena Zhou. Department of General Surgery, Tongde Hospital of Zhejiang Province, 234 Gucui Road, Hangzhou 310012 , China. \\ Email: zyn9072624@sina.com.
}

\begin{abstract}
Background: The present study aimed to investigate the cause-specific survival (CSS) of very small rectal cancer in the context of preoperative serum carcinoembryonic antigen (CEA) elevation.

Methods: Patients diagnosed with node-negative rectal cancer from the Surveillance, Epidemiology, and End Results (SEER) database from January 2004 to December 2010 meeting the inclusion criteria were identified for this study. The Cox proportional hazards regression analyses were conducted to identify independent factors associated with CSS. Pearson's chi-squared tests and Kaplan-Meier methods were performed.

Results: A total of 8,413 patients were included into our study. Kaplan-Meier analyses showed lower 7-year CSS rate of very small tumors $(\leq 5 \mathrm{~mm})$ compared to those larger than $40 \mathrm{~mm}(70.4 \% \mathrm{vs} .76 .0 \%, \log$ rank $\mathrm{P}=0.469)$. Multivariate Cox analyses showed that patients with very small tumor size $(\leq 5 \mathrm{~mm})$ was also associated with a significantly increased risk of cancer-specific mortality compared with those with large tumor size ( $\mathrm{HR}=2.567,95 \% \mathrm{CI}: 1.285$ to $5.130, \mathrm{P}=0.008$, using $\geq 41 \mathrm{~mm}, \mathrm{C}+$ as a reference).

Conclusions: Very small tumor size in the context of preoperative serum CEA elevation could be a surrogate for biological aggressiveness. Our finding would provide a better understanding of tumor biology for us and elicit more future biological researches.
\end{abstract}

Keywords: Tumor size; carcinoembryonic antigen (CEA); rectal cancer

Submitted May 10, 2019. Accepted for publication Aug 08, 2019.

doi: $10.21037 /$ atm.2019.08.81

View this article at: http://dx.doi.org/10.21037/atm.2019.08.81

\section{Introduction}

Rectal cancer is one of the most commonly diagnosed cancers among men and women (1). There were an estimated 43,030 new diagnoses of rectal cancer in 2018 in the US alone (2). Compared with colon cancers, the distinctive anatomic and physiologic features conferred upon rectal diseases higher risk of local spread and recurrence (3). The prognosis of rectal cancer depends on multiple patient and tumor characteristics, including $\mathrm{T}$ stage, lymph node involvement, distant metastases, age, the serum carcinoembryonic antigen (CEA) level of and so on (3-5).
As a $201 \mathrm{kDa}$ highly glycosylated antigen, serum CEA is the single most important tumor marker and elevated preoperative CEA correlate with worse prognosis in rectal cancer (5-7). In 2000, the Colorectal Working Group of the American Joint Committee on Cancer (AJCC) even proposed the inclusion of serum level of CEA (C-stage) into conventional TNM staging of rectal cancer (8). Furthermore, the American Society of Clinical Oncology (ASCO) (6) and the European Group on Tumor Markers (5) have both recommended the use of preoperative serum CEA as a prognostic tool in rectal cancer.

Traditionally, cancer gains the ability to metastasize as 
it grows to a larger size (9). However, recent studies have suggested that metastasis can occur at an early point of tumor progression (10-13). Moreover, CEA-producing tumors have been demonstrated to have a higher ability for metastasis in mouse models $(14,15)$. Then, we have the assumption that very small tumor with elevated serum CEA may suggest early acquisition of metastatic potential.

To the best of our knowledge, no research was reported to address the relationship of tumor size and survival rectal cancer in predicting cause-specific survival (CSS) with elevated serum CEA. In the present study, we sought to investigate the possible interaction between tumor size and elevated serum CEA. Because our hypothesis was that very small tumor size with elevated CEA would present aggressive biology, particularly, we would examine whether very small rectal cancer in the context of CEA elevation was associated with poor prognosis.

\section{Methods}

\section{Study design and data source}

Patients of our study were identified from the Surveillance, Epidemiology, and End Results (SEER) Program of the United States National Cancer Institute, released in 2018. The SEER program collects patient demographic information, cancer diagnostic information, and outcomes in areas participating in the SEER Program, encompassing approximately $28 \%$ of the US population. It does not contain any identifiers and is publicly available for the studies of cancer-based epidemiology. The National Cancer Institute's SEER-Stat software (Surveillance Research Program, National Cancer Institute SEER-Stat software (version 8.3.5) was used to get access to SEER database in this study.

Shown as Figure 1, patients diagnosed with pathological stage I-II rectal cancer treated with surgery between January 1, 2004 and December 31, 2010 were identified from the SEER database. We identified patients diagnosed within these years as pretreatment serum CEA information was recorded starting from 2004 and we wanted to allow for 5 years of follow-up (SEER follow-up ended in 2015).

The exclusion criteria were as follows: tumor size unknown; race unknown; lack of positive histological confirmation; non-adenocarcinoma histologies; pathological $\mathrm{T}$ stage or pathological $\mathrm{N}$ stage unknown; not active followup and lymph-node positive. We also excluded patients without surgery to ensure accurate assessment of tumor size.

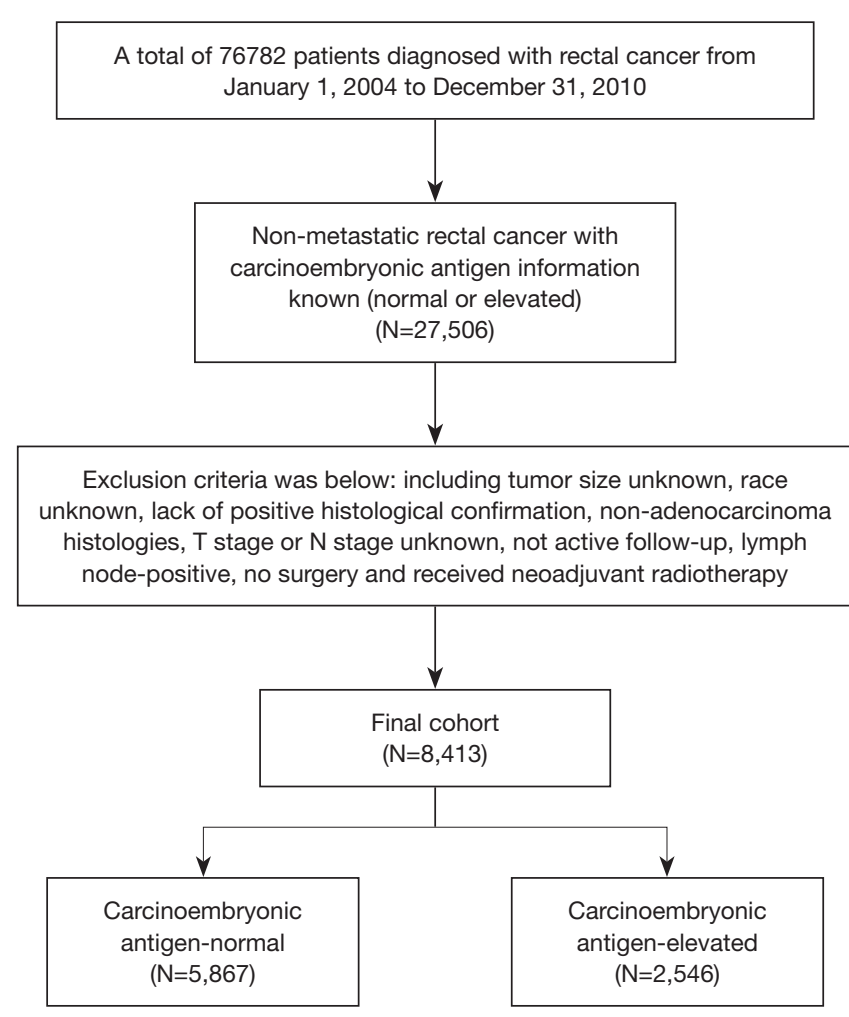

Figure 1 Schematic representation of patient population selected from SEER database. SEER, Surveillance, Epidemiology, and End Results.

The aforementioned process was illustrated in a simplified sequence flow diagram in Figure 1. The whole population was then divided into 5 groups according to tumor size: (I) $\leq 5 \mathrm{~mm}$ (including microscopic focus); (II) $6-20 \mathrm{~mm}$; (III) 21-30 mm; (IV) $31-40 \mathrm{~mm}$ and (V) $\geq 41 \mathrm{~mm}$.

\section{Statistical analyses}

In our study, different clinicopathologic factors between the CEA-normal and CEA-elevated groups were compared using Pearson's chi-squared tests. The study endpoint used in our study was CSS. The cause of death was categorized as rectal cancer specific or non-rectal cancer related, and CSS was calculated from the date of diagnosis to the date of rectal cancer death. Patients who died of other causes were censored at the date of death. A variable combined with tumor size and CEA levels was then defined to evaluate the interaction between tumor size and CEA level in predicting CSS. The CSS curves of our study were constructed using the Kaplan-Meier method, and log-rank tests were used to 
determine a univariate survival difference. Furthermore, we also conducted pair-wise comparisons between different combinations of tumor size and CEA level to determine the presence of significant CSS differences. Cox proportional hazard models were constructed to adjust hazard ratios (HRs) with 95\% confidence intervals (CIs). Statistical analysis was mainly performed using SPSS version 22 (SPSS Inc., IL, USA); and two-sided $\mathrm{P}<0.05$ was considered statistically significant.

\section{Results}

\section{Baseline characteristics}

A total of 8,413 patients diagnosed with pathological stage I-II rectal cancer were identified from the SEER database. Median follow-up time among censored patients was 84 months, and 1,144 (13.6\%) patients died of rectal cancer at the end of the follow-up time. Of all, 5,867 patients $(69.7 \%)$ were stratified into the CEA-normal group, and 2,546 patients $(30.3 \%)$ were stratified into the CEA-elevated group. The elevation of CEA was correlated with higher T stage, older age, black, mucinous adenocarcinoma or signet ring cell carcinoma and larger tumor size $(\mathrm{P}<0.001$, Table 1). Within the smallest size stratum $(\leq 5 \mathrm{~mm}), 211$ patients (3.6\%) had CEA-normal disease and 37 patients (1.5\%) had CEA-elevated disease.

\section{Interaction between tumor size and CEA levels in CSS}

Multivariate Cox analysis was conducted after adjusting for pathological $\mathrm{T}$ stage, year of diagnosis, age at diagnosis, race, gender, tumor location, tumor grade, histology (Table 2). The results of multivariate analysis showed a significant interaction between tumor size and CEA levels in determining CSS $(\mathrm{P}<0.001$, Table 2). Furthermore, the results also indicated more favorable prognosis of lower pathological $\mathrm{T}$ stage, younger age, white race, females, lower tumor grade and adenocarcinoma histology (Table 2). Separate Kaplan-Meier CSS curves for CEA-normal and CEA-elevated patients stratified by categorical tumor size are provided in Figure 2A,B. Among CEA-elevated (C+) cases, Kaplan-Meier analysis showed 7-year CSS (median follow-up time among censored patients was 7 years) decreased as tumor enlarged except when tumor size was less than $5 \mathrm{~mm}$. We found that very small tumors $(\leq 5 \mathrm{~mm})$ had poorer 7-year CSS compared to those larger than $40 \mathrm{~mm}$ (70.4\% vs. $76.0 \%)$, but it did not achieve significant difference $(\log$-rank $\mathrm{P}=0.469)$, which was probably because of the relatively small sample size of group $(\leq 5 \mathrm{~mm}, \mathrm{C}+)$ $(\mathrm{n}=37$, Figure $2 A)$. Even after controlling for multiple patient-specific factors, however, patients with very small tumor size $(\leq 5 \mathrm{~mm})$ was associated with a significantly increased risk of cancer-specific mortality compared with those with large tumor size ( $\mathrm{HR}=2.567,95 \% \mathrm{CI}: 1.285$ to $5.130, \mathrm{P}=0.008$, using $\geq 41 \mathrm{~mm}, \mathrm{C}+$ as a reference, Table 2). In addition, we have also conducted the KaplanMerier CSS analyses of serum CEA levels and tumor size, respectively (Figures $S 1, S 2$ ). The results showed that serum CEA elevation and larger tumor are associated with worse prognosis.

In contrast, among patients with normal level of CEA, tumor size correlated directly with CSS and 7-year CSS gradually decreased as tumor size increased, without exception (log-rank $\mathrm{P}=0.002$, Figure $2 B$ ). Similar phenomenon was found after controlling for $\mathrm{T}$ stage, year of diagnosis, age at diagnosis, race, gender, tumor location, tumor grade, histology chemotherapy (Table 2).

The relationship of adjusted HRs and different tumor size groups with elevated or normal level of CEA was visualized in Figure 3, in which the highest adjusted HR of very small tumor size $(\leq 5 \mathrm{~mm})$ with elevated level of CEA was very prominent.

\section{Discussion}

In the present study, aimed to investigate the possible interaction of tumor size and elevated serum CEA in predicting CSS of node-negative rectal cancer (stage I-II), we provide the first evidence that very small rectal cancers $(\leq 5 \mathrm{~mm})$ were associated with approximately double risk of cancer-specific mortality compared with tumors sized 6-20, $21-30,31-40$, or $\geq 41 \mathrm{~mm}$ in the context of serum CEAelevation, even after adjusting for potential explanatory factors such as $\mathrm{T}$ stage, year of diagnosis, age at diagnosis, race, gender, tumor location, tumor grade, histology and chemotherapy. This finding was consistent with our previous hypothesis that very small tumor size with elevated CEA would be associated with aggressive biology. On the other hand, among those with normal serum CEA, the risk of mortality increased monotonically with tumor enlarging.

In 2011, an impressive research found that very small tumors would present aggressive biology when involved in node-positive breast cancer (16). Then, similar findings were demonstrated in colon and prostate cancer $(17,18)$. 
Table 1 Comparison of baseline characteristics by the CEA level in SEER cohort

\begin{tabular}{|c|c|c|c|}
\hline Variable & \multicolumn{2}{|c|}{ No. of patients (\%) } & $\mathrm{P}$ \\
\hline T stage & & & $<0.001$ \\
\hline $\mathrm{T} 1$ & $1,587(27.0)$ & $297(11.7)$ & \\
\hline $\mathrm{T} 2$ & $1,996(34.0)$ & $646(25.4)$ & \\
\hline $\mathrm{T} 4$ & $183(3.1)$ & $245(9.6)$ & \\
\hline Year of diagnosis & & & 0.086 \\
\hline 2004-2007 & 3,401 (58.0) & $1,527(60.0)$ & \\
\hline 2008-2010 & $2,466(42.0)$ & $1,019(40.0)$ & \\
\hline$>65$ & $3,090(52.7)$ & $1,538(60.4)$ & \\
\hline Race & & & $<0.001$ \\
\hline White & 4,939 (84.2) & 2,019 (79.3) & \\
\hline Black & $423(7.2)$ & 268 (10.5) & \\
\hline Other & 505 (8.6) & 259 (10.2) & \\
\hline Gender & & & 0.466 \\
\hline Male & $3,272(55.8)$ & 1,398 (54.9) & \\
\hline Female & 2,595 (44.2) & $1,148(45.1)$ & \\
\hline Grade I/II & $5,066(86.3)$ & 2,192 (86.1) & \\
\hline Grade III/IV & $622(10.6)$ & $290(11.4)$ & \\
\hline Unknown & $179(3.1)$ & $64(2.5)$ & \\
\hline Histology & & & $<0.001$ \\
\hline Adenocarcinoma & $5,711(97.3)$ & 2,383 (93.6) & \\
\hline Mucinous adenocarcinoma/signet ring cell carcinoma & $156(2.7)$ & $163(6.4)$ & \\
\hline Tumor size (mm) & & & $<0.001$ \\
\hline$\leq 5$ & $211(3.6)$ & $37(1.5)$ & \\
\hline $6-20$ & $1,354(23.1)$ & $286(11.2)$ & \\
\hline $21-30$ & $1,293(22.0)$ & $379(14.9)$ & \\
\hline $31-40$ & $1,160(19.8)$ & $477(18.7)$ & \\
\hline$\geq 41$ & $1,849(31.5)$ & $1,367(53.7)$ & \\
\hline
\end{tabular}

CEA, carcinoembryonic antigen; SEER, Surveillance, Epidemiology, and End Results. 
Table 2 Multivariate Cox regression analyses of CSS in SEER cohort

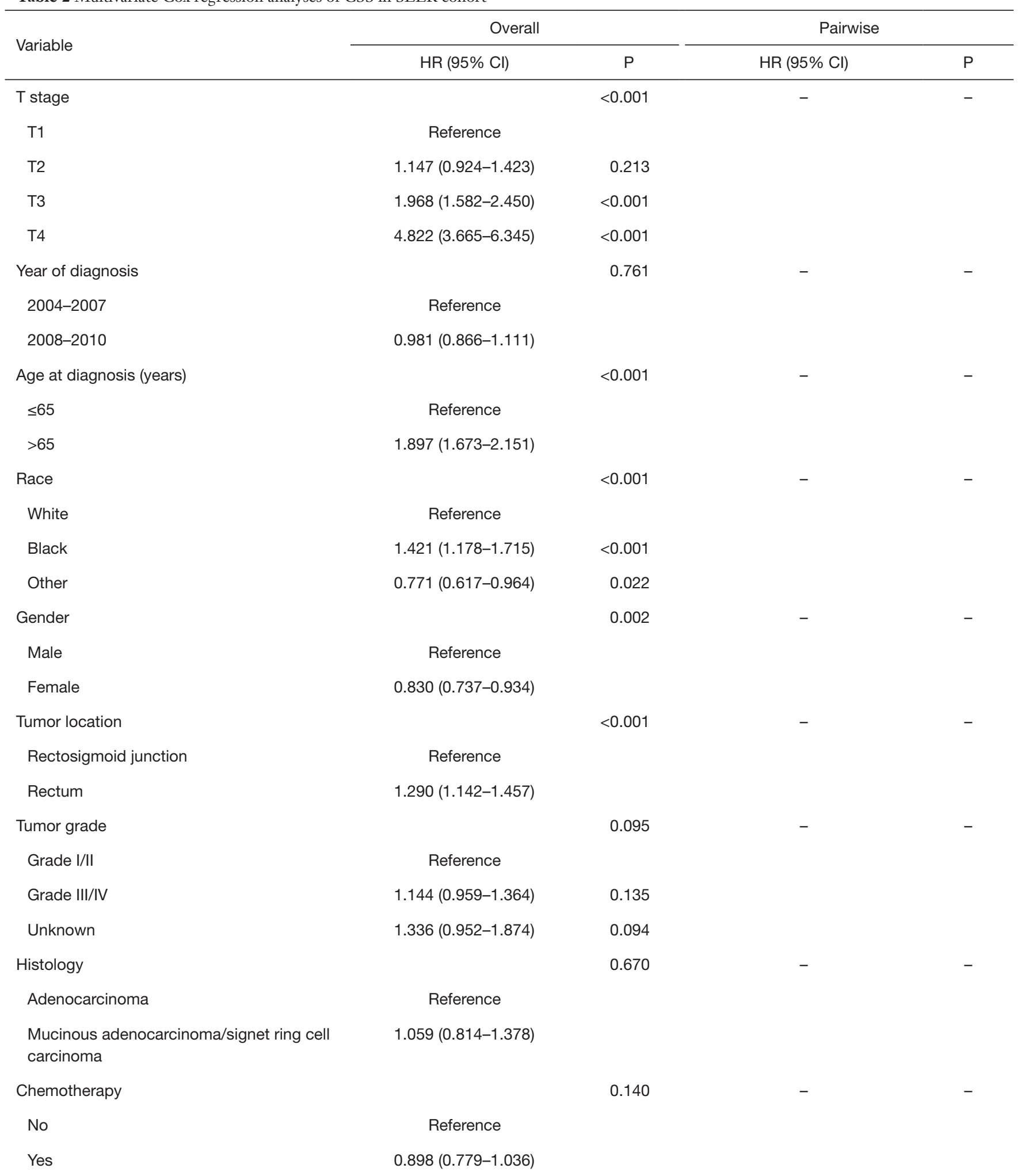

Table 2 (continued) 
Table 2 (continued)

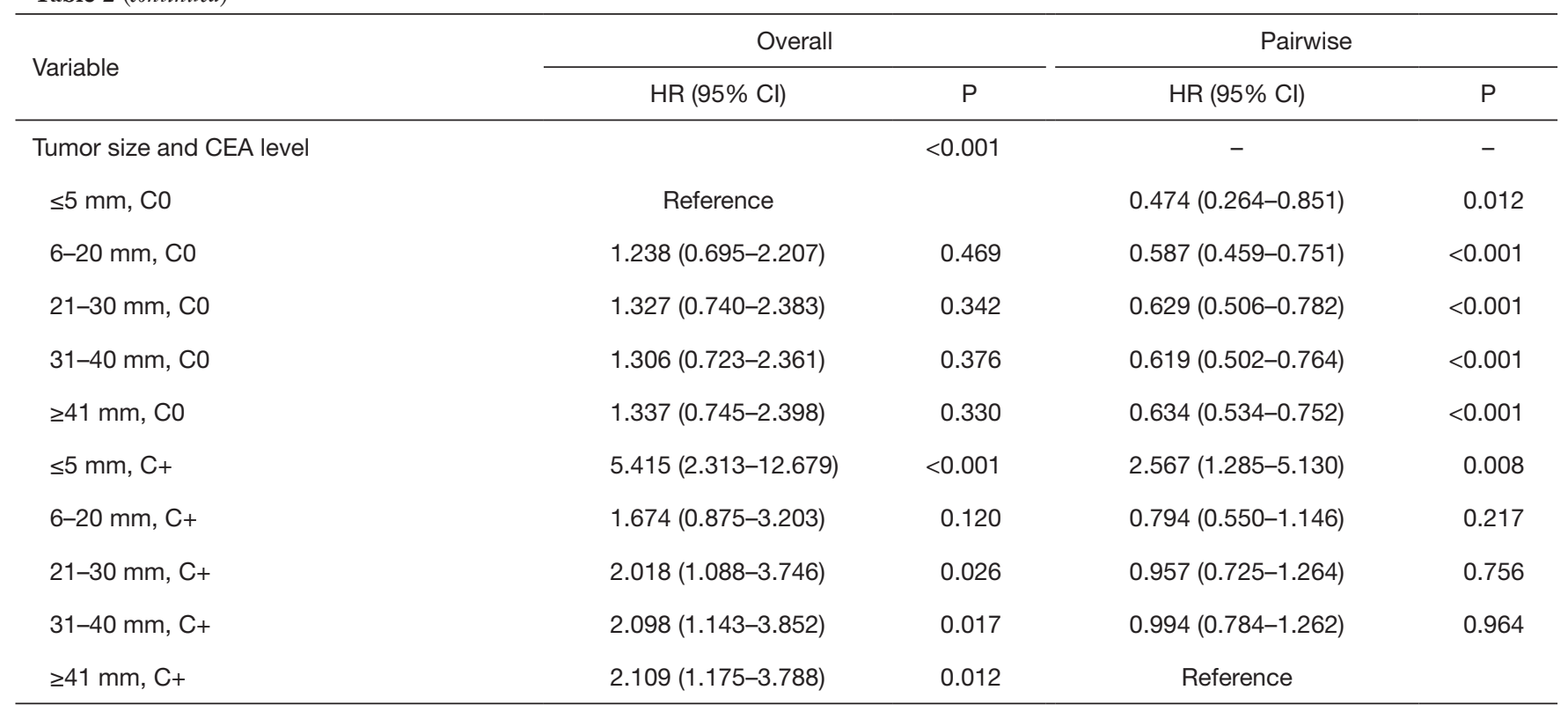

CO, CEA-normal; C+, CEA-elevated. CSS, cause-specific survival; SEER, Surveillance, Epidemiology, and End Results; HR, hazard ratio; $\mathrm{Cl}$, confidence interval.
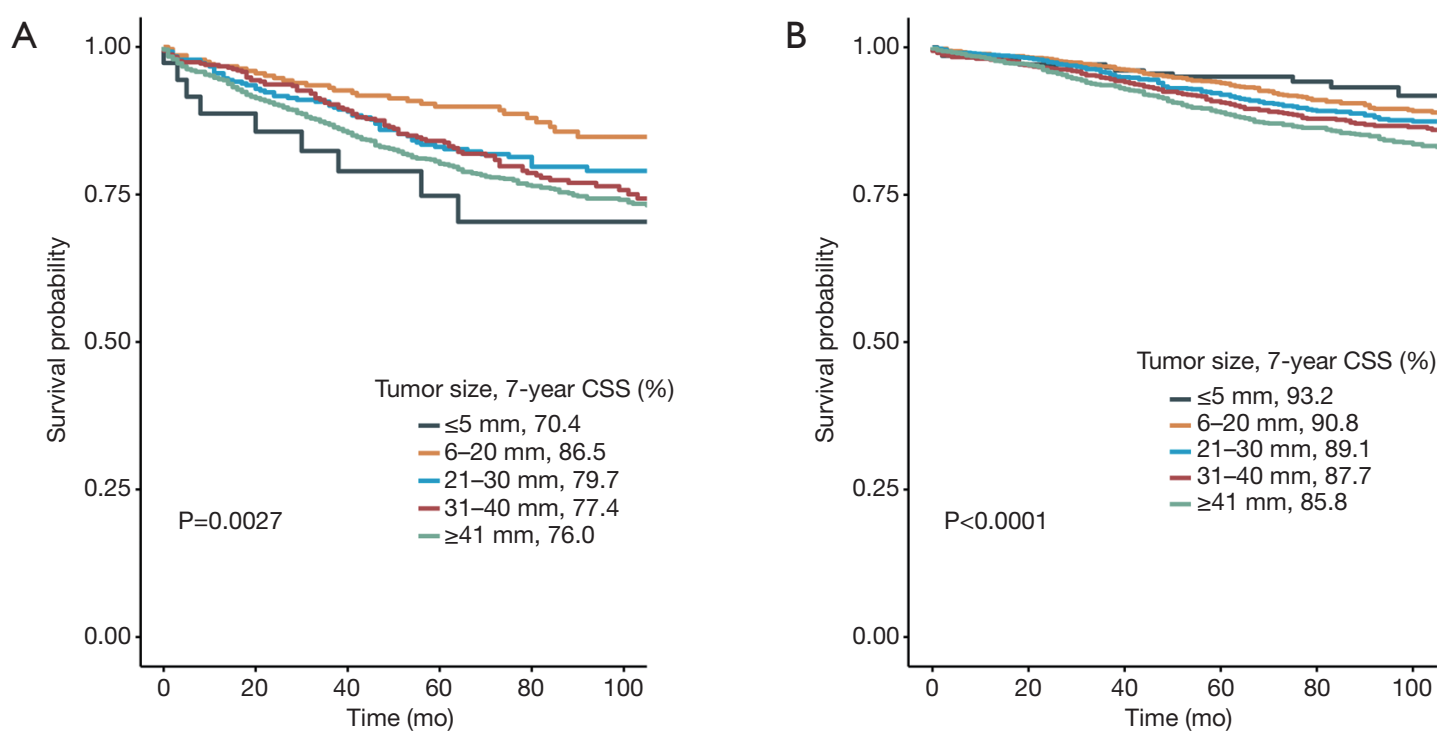

Figure 2 Kaplan-Meier CSS curves stratified by categorical tumor size in: (A) serum CEA-elevated rectal cancer; (B) serum CEA-normal rectal cancer. CSS, cause-specific survival; CEA, carcinoembryonic antigen.

These findings challenged the traditional view that cancer gains the ability to metastasize as it grows to a larger size (9). Among many tumor markers and prognostic indicators, the serum CEA was the single most important one and played an irreplaceable role in colorectal cancer $(3,5)$. And
CEA-producing tumors have long been demonstrated to have a higher ability for metastasis $(14,15)$. We then have the appropriate assumption that rectal cancer with very small primary lesions, when involved in serum CEAelevation, might be a surrogate for biologically aggressive 


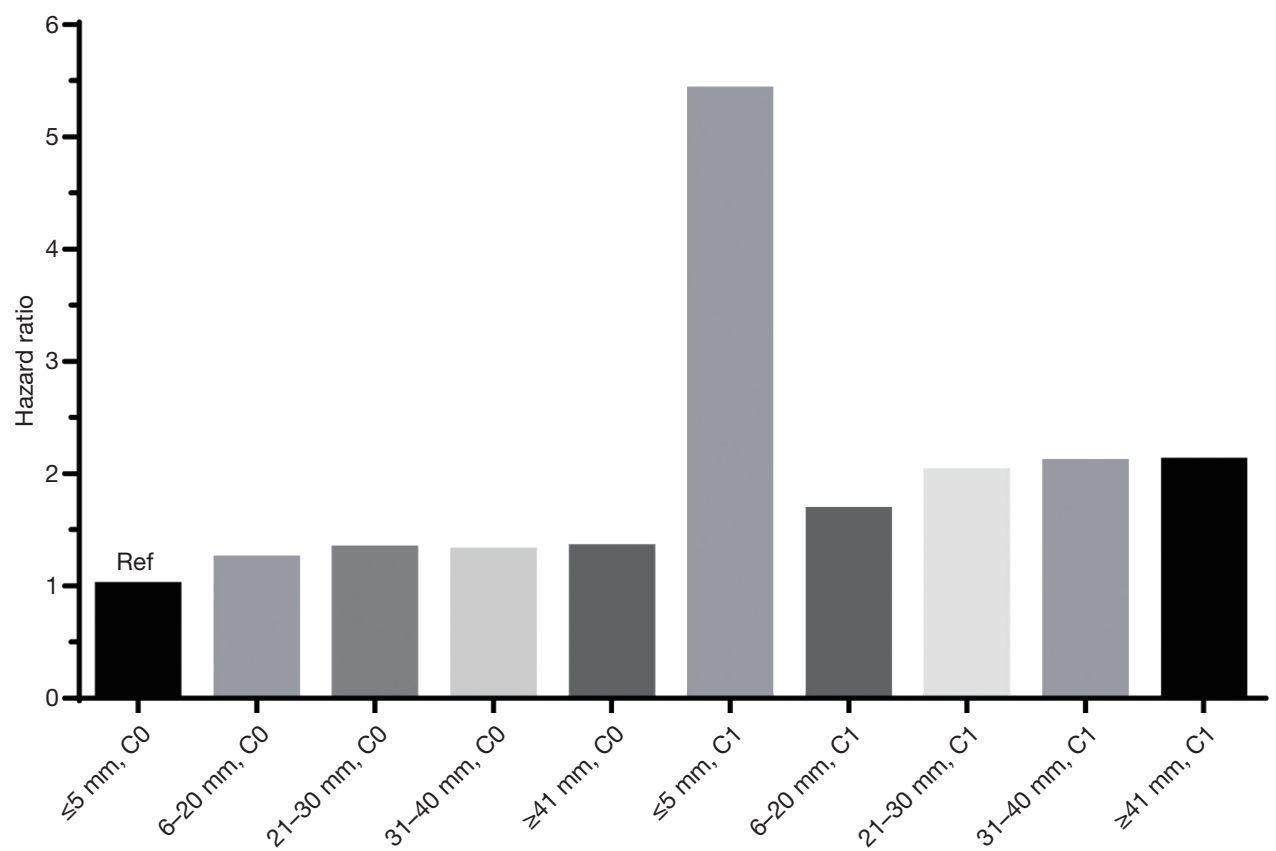

Figure 3 Adjusted hazard ratios comparing CSS among different tumor size and serum CEA levels groups. CSS, cause-specific survival; CEA, carcinoembryonic antigen.

disease, where metastases pathways had more advantage than those resulting in "reattachment in or at the primary site" according to the "self-seeding" concept (9), and cancer cells experienced more clonal expansions and mutations according to the linear progression model of cancer progression (11), contributing to the significantly worse prognosis.

Our finding would have important implications for both researchers and clinicians. On the one hand, the present study could provide better understanding of rectal cancer biology to elicit further researches. CSS differences were presented both before and after adjusting for other known prognostic factors in rectal cancer. The dissemination of very small serum CEA-elevated rectal cancer cells might occur early after acquisition of fully malignant traits and these tumor cells are also thought to share most of their characteristics with the primary tumor (11). Very small serum CEA-elevated rectal disease could be a surrogate of increased biological aggressiveness and experience more clonal expansions and mutations which deserve future identifications and might elicit the discovery of new genomic changes or new drug targets in further researches (11).

On the other hand, according to the National Comprehensive Cancer Network (NCCN) guidelines, stage I rectal cancer was treated with surgical resection alone and stage II disease was treated with neoadjuvant chemoradiation therapy with subsequent surgical resection and systemic chemotherapy as the current standard of care for the intended cure of rectal cancer $(19,20)$. As is known, many previous studies showed that larger tumors are associated with poorer oncologic outcomes (21-25). While tumor size appeared to be of minor value within the rectal disease (26), in our study, very small rectal cancer $(\leq 5 \mathrm{~mm})$ presented $156.7 \%$ increased risk of rectal cancer-specific mortality compared with large rectal disease $(\geq 41 \mathrm{~mm})$ with involvement of elevated serum CEA, even after adjusting for other prognostic factors. Our present results indicated that very small stage II rectal cancer might be candidates for intensification of chemoradiation therapy and chemoradiation therapy should also be considered for very small stage I rectal disease in the context of serum CEA-elevation. With the widely accept and application of pretreatment serum CEA examination and colonoscopy (3), in especial, the main strength of our study to guide clinical treatment was prior to the operation, when neoadjuvant therapy should be applied or paid more attention based on the results of blood examination (elevated serum CEA) and colonoscopy (very small tumor size).

While limited to very small sample size of tumor size less than $5 \mathrm{~mm}$ with elevated serum CEA ( $\mathrm{n}=37)$, we were not 
able to effectively study the role of chemoradiation therapy, our present results might still more or less shed some enlightenment for clinicians to counsel patients with very small serum CEA-elevated rectal cancer about prognosis and guide clinical treatment.

The results of our multivariate analysis supported the finding that blacks were associated with higher risk of mortality compared with white patients (27). In Pearson's chi-squared tests, blacks were more likely to present elevated serum CEA compared with whites, which might be one reason of blacks' poorer prognosis. Furthermore, higher $\mathrm{T}$ stage, older age, mucinous adenocarcinoma or signet ring cell carcinoma and larger tumor size were more prone to serum CEA elevation. Our study also supported the excellent prognosis for patients with very small tumors in the context of normal serum CEA with an unadjusted 7 -year CSS of $93.2 \%$.

This study had some limitations. First, it did not include multiple potential prognostic factors such as microsatellite instability, BRAF V600E mutation, radiotherapy and CEA level, introducing biases to some extent (28-30). Second, the sample size is relatively small. In especial, we had very few of patients within our smallest size group, and they represented a very small percentage of the overall cohort. This limitation was inherent to the study design given the relative rarity of very small rectal cancers with elevated serum CEA. Although this limitation might therefore reduce the general applicability of our findings, future researches into the biological characteristics of very small rectal disease with the involvement of serum CEA-elevation could potentially benefit all patients with rectal cancer. Third, because of the limitation of the SEER database, we cannot conduct concerning the detailed value of serum CEA, including the association of CEA and tumor size both as continuous variable. Finally, the present study was retrospective rather than prospective. These findings still need to be validated in other prospective cohorts. In spite of these limitations, the SEER database provides the opportunity for us to analyze a large sample size of patients with active follow-up. Our data represents the most robust evaluation of interaction between tumor size and elevated serum CEA.

In conclusion, our study provided the first evidence that very small tumors with elevated serum CEA might present significantly increased risk of mortality compared with larger tumors, suggesting that very small tumor size in the context of serum CEA-elevation could be a surrogate for biological aggressiveness that was not explained by known patient and tumor characteristics such as the depth of tumor invasion or tumor grade. Our finding, if validated in other databases, further researches should focus on the subset of very small rectal cancer with serum CEA-elevation to identify genetic or molecular changes that contribute to elevation of serum CEA and thus increasing the risk of mortality; in clinic, more attention of chemoradiation therapy should be paid to very small rectal cancer with serum CEA-elevation, especially prior to the operation based on the results of blood examination and colonoscopy.

\section{Acknowledgments}

None.

\section{Footnote}

Conflicts of Interest: The authors have no conflicts of interest to declare.

Ethical Statement: This study was based on the publicly available SEER database and was approved by the Ethical Committee and Institutional Review Board of Tongde Hospital. The authors are accountable for all aspects of the work in ensuring that questions related to the accuracy or integrity of any part of the work are appropriately investigated and resolved.

\section{References}

1. Siegel RL, Miller KD, Fedewa SA, et al. Colorectal cancer statistics, 2017. CA Cancer J Clin 2017;67:104-17.

2. Siegel RL, Miller KD, Jemal A. Cancer statistics, 2018. CA Cancer J Clin 2018;68:11.

3. Goldenberg BA, Holliday EB, Helewa RM, et al. Rectal Cancer in 2018: A Primer for the Gastroenterologist. Am J Gastroenterol 2018;113:1763-71.

4. Frederick LGMD, David LPMD, Irvin DFMD, et al. AJCC Cancer Staging Manual. Springer, 2010.

5. Duffy MJ, Van DA, Haglund C, et al. Tumour markers in colorectal cancer: European Group on Tumour Markers (EGTM) guidelines for clinical use. Eur J Cancer 2007;43:1348-60.

6. Locker GY, Hamilton S, Harris J, et al. ASCO 2006 update of recommendations for the use of tumor markers in gastrointestinal cancer. J Clin Oncol 2006;24:5313-27.

7. Hammarström S. The carcinoembryonic antigen (CEA) family: structures, suggested functions and expression 
in normal and malignant tissues. Semin Cancer Biol 1999;9:67-81.

8. Compton C, Fenoglio-Preiser CM, Pettigrew N, et al. American Joint Committee on Cancer prognostic factors consensus conference. Cancer 1999;86:2436.

9. Norton L, Massagué J. Is cancer a disease of self-seeding? Nature Medicine 2006;12:875-8.

10. $\mathrm{Hu} \mathrm{Y,} \mathrm{Yu} \mathrm{X,} \mathrm{Xu} \mathrm{G,} \mathrm{et} \mathrm{al.} \mathrm{Metastasis:} \mathrm{an} \mathrm{early} \mathrm{event} \mathrm{in} \mathrm{cancer}$ progression. J Cancer Res Clin Oncol 2016;143:1-13.

11. Klein CA. Parallel progression of primary tumours and metastases. Nat Rev Cancer 2009;9:302-12.

12. Popiolek, Andren, Andersson, et al. Natural History of Early, Localized Prostate Cancer: A Final Report from Three Decades of Follow-up. Eur Urol 2013;63:428-35.

13. Pavlidis N, Khaled H, Gaafar R. A mini review on cancer of unknown primary site: a clinical puzzle for the oncologists. J Adv Res 2015;6:375-82.

14. Jessup JM, Giavazzi R, Campbell D, et al. Growth potential of human colorectal carcinomas in nude mice: association with the preoperative serum concentration of carcinoembryonic antigen in patients. Cancer Res 1988;48:1689-92.

15. Tibbetts LM, Doremus CM, Tzanakakis GN, et al. Liver metastases with 10 human colon carcinoma cell lines in nude mice and association with carcinoembryonic antigen production. Cancer 1993;71:315-21.

16. Wo JY, Chen K, Neville BA, et al. Effect of Very Small Tumor Size on Cancer-Specific Mortality in NodePositive Breast Cancer. J Clin Oncol 2011;29:2619.

17. Muralidhar V, Nipp RD, Ryan DP, et al. Association Between Very Small Tumor Size and Increased CancerSpecific Mortality in Node-Positive Colon Cancer. Dis Colon Rectum 2016;59:187-93.

18. Muralidhar V, Mahal BA, Nezolosky MD, et al. Association between very small tumour size and increased cancerspecific mortality after radical prostatectomy in lymph node-positive prostate cancer. BJU Int 2016;118:279-85.

19. Edge SB, Compton CC. The American Joint Committee on Cancer: the 7th edition of the AJCC cancer staging manual and the future of TNM. Ann Surg Oncol

Cite this article as: Pan H, Cui J, Cai K, Zhou Y. Increased cancer-specific mortality of very small size in carcinoembryonic antigen-elevated rectal cancer. Ann Transl Med 2019;7(18):447. doi: 10.21037/atm.2019.08.81
2010;17:1471-4.

20. Kolarich A, George TJ Jr, Hughes SJ, et al. Rectal cancer patients younger than 50 years lack a survival benefit from NCCN guideline-directed treatment for stage II and III disease. Cancer 2018;124:3510-9.

21. Vollmer RT. Percentage of tumor and tumor length in prostate biopsy specimens: a study of American veterans. Am J Clin Pathol 2008;130:940.

22. Vollmer RT. Percentage of Tumor in Prostatectomy SpecimensA Study of American Veterans. Am J Clin Pathol 2009;131:86.

23. Saha S, Shaik M, Johnston G, et al. Tumor size predicts long-term survival in colon cancer: an analysis of the National Cancer Data Base. Am J Surg 2015;209:570-4.

24. Fukui T, Fukumoto K, Okasaka T, et al. Prognostic impact of tumour size in completely resected thymic epithelial tumours. Eur J Cardiothorac Surg 2016;50:1068-74.

25. Shibing Y, Liangren L, Qiang W, et al. Impact of tumour size on prognosis of upper urinary tract urothelial carcinoma after radical nephroureterectomy: a multi-institutional analysis of 795 cases. BJU Int 2016;118:902-10.

26. Kornprat P, Pollheimer MJ, Lindtner RA, et al. Value of tumor size as a prognostic variable in colorectal cancer: a critical reappraisal. Am J Clin Oncol 2011;34:43.

27. Joseph DA, Johnson CJ, White A, et al. Rectal cancer survival in the United States by race and stage, 2001 to 2009: Findings from the CONCORD-2 study. Cancer 2017;123 Suppl 24:5037-58.

28. Lochhead P, Kuchiba A, Imamura Y, et al. Microsatellite Instability and BRAF Mutation Testing in Colorectal Cancer Prognostication. J Natl Cancer Inst 2013;105:1151-6.

29. Staab HJ, Anderer FA, Brummendorf T, et al. Prognostic significance of preoperative carcinoembryonic antigen in stomach and colorectal cancer. Cancer Detect Prev 1983;6:149.

30. Thirunavukarasu P, Sukumar S, Sathaiah M, et al. C-stage in Colon Cancer: Implications of Carcinoembryonic Antigen Biomarker in Staging, Prognosis, and Management. J Natl Cancer Inst 2011;103:689-97. 


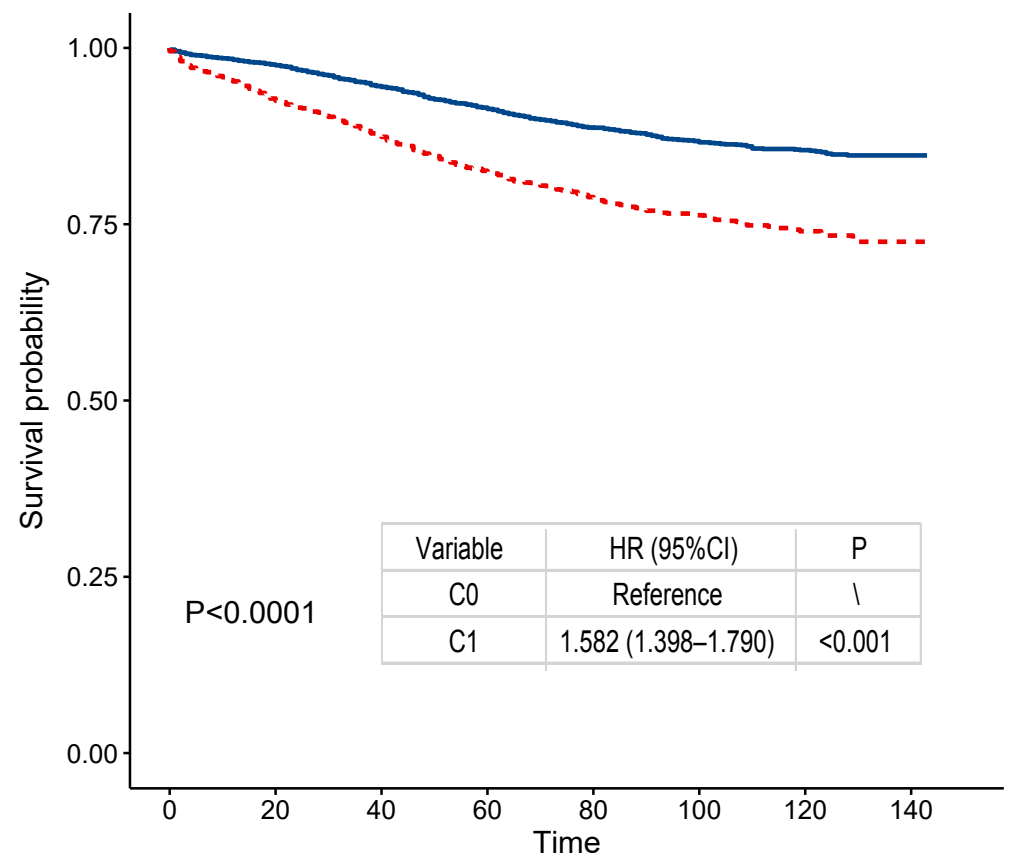

Number at risk

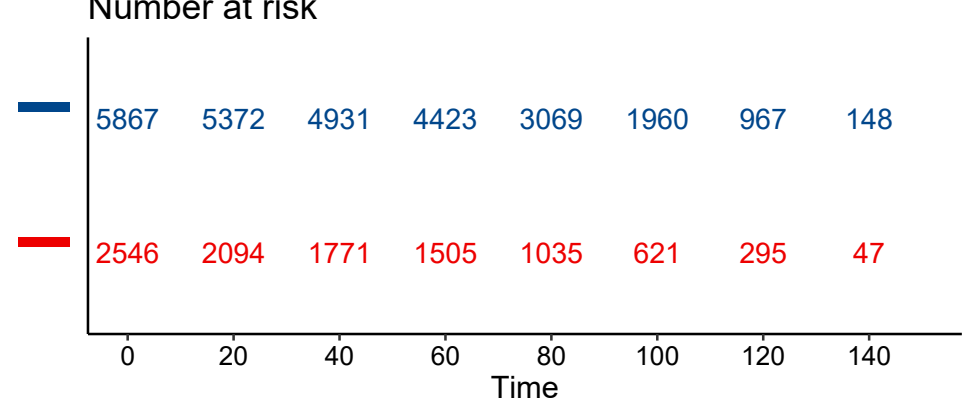

Number of censoring

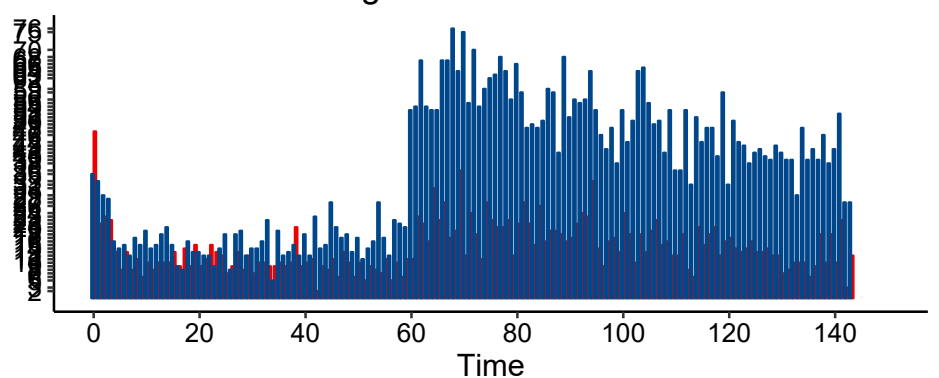

Figure S1 Kaplan-Meir CSS curves according to serum CEA levels in the whole cohort. CSS, cause-specific survival; CEA, carcinoembryonic antigen. 


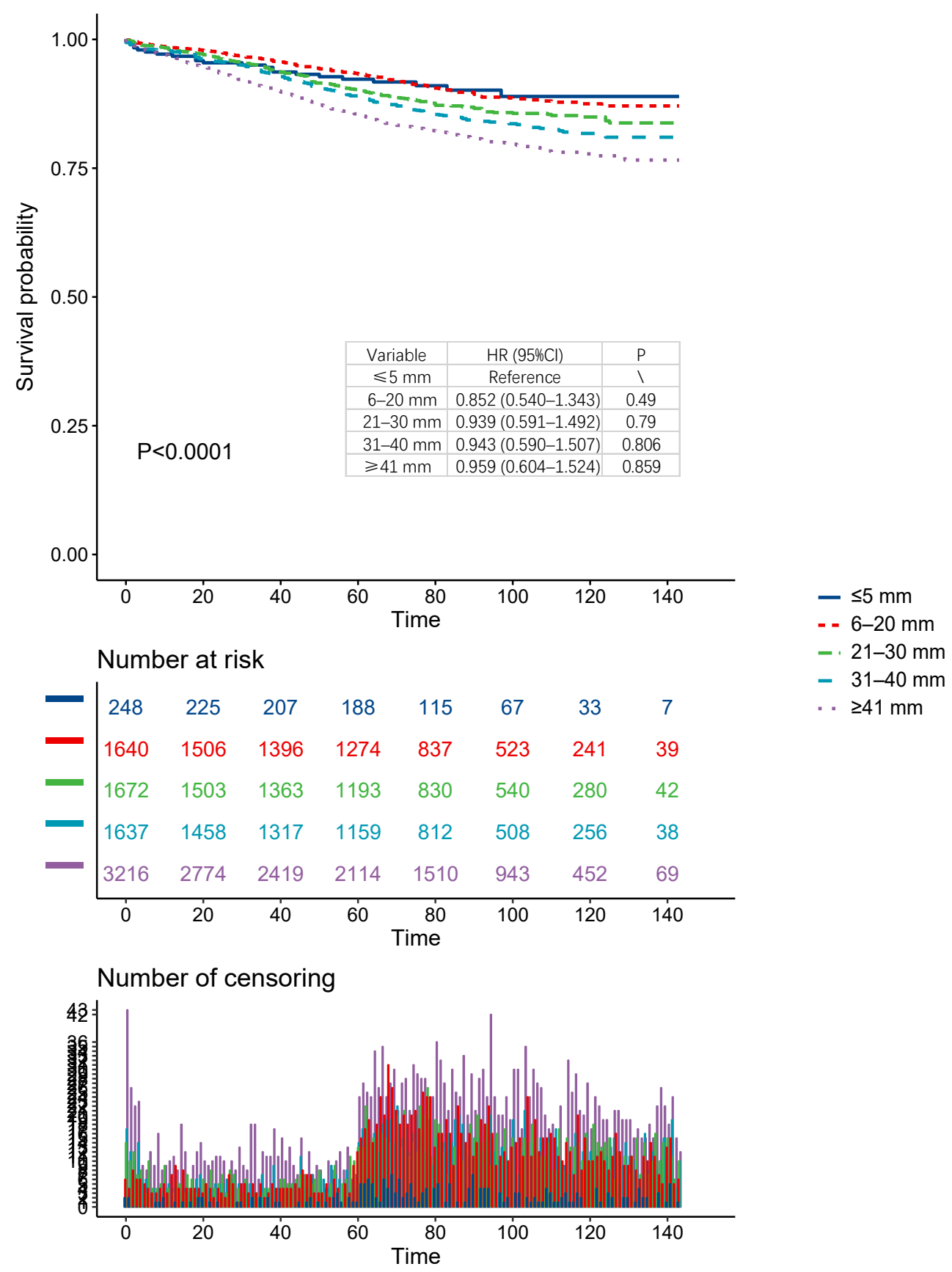

Figure S2 Kaplan-Meir CSS curves according to tumor size in the whole cohort. CSS, cause-specific survival. 